Purdue University Purdue e-Pubs

RCHE Publications

Regenstrief Center for Healthcare Engineering

5-24-2006

\title{
The need for organizational change in patient safety initiatives
}

James G. Anderson

Purdue University, andersonj@purdue.edu

Follow this and additional works at: http://docs.lib.purdue.edu/rche_rp

Anderson, James G., "The need for organizational change in patient safety initiatives" (2006). RCHE Publications. Paper 21.

http://docs.lib.purdue.edu/rche_rp/21

This document has been made available through Purdue e-Pubs, a service of the Purdue University Libraries. Please contact epubs@purdue.edu for additional information. 


\title{
The need for organizational change in patient safety initiatives
}

\author{
James G. Anderson ${ }^{a, *}$, Rangaraj Ramanujam ${ }^{a}$, Devon Hensel ${ }^{b}$, \\ Marilyn M. Anderson ${ }^{c}$, Carl A. Sirio ${ }^{d}$ \\ a Purdue University, West Lafayette, IN 47907, United States \\ ${ }^{\mathrm{b}}$ Indiana University School of Medicine, Indianapolis, IN 46202, United States \\ c Anderson Consulting, West Lafayette, IN 47907, United States \\ d University of Pittsburgh, United States
}

\section{A R T I C L E I N F O}

\section{Article history:}

Received 21 October 2005

Received in revised form

8 February 2006

Accepted 24 May 2006

Keywords:

Medical errors

Incident reporting systems

Organizational change

\begin{abstract}
A B S T R A C T
Objectives: This study describes a computer simulation model that has been developed to explore organizational changes required to improve patient safety based on a medication error reporting system.

Methods: Model parameters for the simulation model were estimated from data submitted to the MEDMARX medication error reporting system from 570 healthcare facilities in the U.S. The model's results were validated with data from the Pittsburgh Regional Healthcare Initiative consisting of 44 hospitals in Pennsylvania that have adopted the MEDMARX medication error reporting system. The model was used to examine the effects of organizational changes in response to the error reporting system. Four interventions were simulated involving the implementation of computerized physician order entry, decision support systems and a clinical pharmacist on hospital rounds.

Conclusions: Results of the analysis indicate that improved patient safety requires more than clinical initiatives and voluntary reporting of errors. Organizational change is essential for significant improvements in patient safety. In order to be successful, these initiatives must be designed and implemented through organizational support structures and institutionalized through enhanced education, training, and implementation of information technology that improves work flow capabilities.
\end{abstract}

@ 2006 Elsevier Ireland Ltd. All rights reserved.

\section{Introduction}

The Institute of Medicine (IOM) report, To Err is Human: Building a Safer Health System [1], estimated that between 44,000 and 98,000 deaths occur in the U.S. each year as a result of medical errors. In fact, there is evidence that morbidity and mortality from prescription errors increased between 1983 and 1998 by $243 \%$ [2]. A significant number of these errors involve medi- cations. A meta-analysis of 39 prospective studies indicated that adverse drug reactions may rank between the fourth and seventh leading cause of deaths in the U.S. [3]. One study of medication errors in 36 hospitals and skilled nursing facilities in Georgia and Colorado found that $19 \%$ of the doses were in error; $7 \%$ of the errors could have resulted in adverse drug events [4]. Recognizing the magnitude of the medication error problem, a subsequent IOM report, Crossing the Quality Chasm:

\footnotetext{
* Corresponding author at: Department of Sociology \& Anthropology, Purdue University, 700 W. State Street, West Lafayette, IN 47907-2059, United States. Tel.: +1 765494 4703; fax: +1 7654961476.

E-mail address: andersonj@cla.purdue.edu (J.G. Anderson). 
A New Health System for the 21st Century [5] recommended that confidential voluntary reporting systems be adopted in all health care organizations. At present the Veteran's Administration, about half of the states, many hospitals and private organizations have developed error reporting systems in an effort to improve patient safety [6].

Traditionally, efforts to reduce errors have focused on training, rules and sanctions. Also, hospitals have relied on voluntary reporting of errors. Currently only $5-10 \%$ of medication errors that result in harm to patients are reported [7]. As a result little progress has been made since the IOM report 5 years ago [8].

\subsection{Error reporting systems}

The first step in reducing medication errors is standardized reporting of the necessary data to understand the nature of the problem. A number of reporting systems have been developed. Some of these systems are voluntary, others mandatory. The most successful systems are modeled after the Aviation Safety Report System (ASRS). This system is anonymous, voluntary and administered by the NASA for the Federal Aviation Administration [9]. The Veterans Administration Patient Safety Reporting System (PSRS) is patterned after the ASRS system $[10,11]$. The Institute for Safe Medical Practice (ISMP) also has developed a voluntary reporting system for medication errors [12]. Another system has been developed by the Food and Drug Administration (FDA) called Data Watch for the reporting of adverse events arising from medications and medical devices [6].

Other reporting systems have been developed for ICUs [13], at the Ohio State University Health Care System [14], and for primary care clinics $[15,16]$. Some states in the U.S. have mandatory medical error reporting databases. However, these systems require the identification of the responsible parties and are used for disciplinary actions [6]. Medical error reporting systems are also being implemented in other countries such as Korea [17], Japan [18] and France [19].

In some instances error reporting systems are being implemented among multiple institutions in order to share learning regarding incidence and types of errors and ways to improve patient safety. For example, Johns Hopkins University designed and implemented a Web-based ICU safety reporting system in 18 ICUs across the United States [20]. The Pittsburgh Regional Health Care Initiative is a consortium of 44 hospitals that share data on nosocomial (hospital acquired) infections and medication errors [21].

This study uses data from the MEDMARX system [22]. The United States Pharmacopoeia (USP) introduced MEDMARX in 1998. It is an internet-accessible, anonymous medication error reporting system. Currently 775 hospitals and health systems use the system. Medication errors are reported in a standard format. A medication error is defined as "... any preventable event that may cause or lead to inappropriate medication use or patient harm while the medication is in the control of the healthcare professional, patient, or consumer. Such events may be related to professional practice; healthcare practice; healthcare products' procedures, and systems including prescribing; order communication; product labeling; packaging, and nomenclature; compounding; dis- pensing; distribution; administration; education; monitoring and use" [23].

\subsection{Organizational actions}

The majority of medical errors result from poorly designed healthcare systems rather than from negligence on the part of health care providers. Successful patient safety initiatives require cognitive, social and organizational changes as well as reporting $[24,25]$. Current health care systems are complex and fragmented, involving interactions among a number of health care professionals with various levels of education and training [26]. The discontinuous nature of patient care fosters errors [27]. Remedying the systemic problems that lead to error will require organizational changes at the point of care [28].

Members of the study team were associated with an Agency for Healthcare Research and Quality (AHRQ) funded research project. The purpose of the project is to achieve sustainable improvements in health care on a regional basis by sharing information. The objective of the project is to improve patient care and safety in over 44 hospitals [21,26]. The hospitals, working in a collaboration called the Pittsburgh Regional Healthcare Initiative (PRHI), have been sharing information about their medication errors for the past 3 years. The overall objective is to leverage the data from reporting to initiate region-wide process improvements. The assumptions underlying this regional reporting system are described using a learning chain model as shown in Fig. 1.

The framework suggests that the link between reporting and learning requires that data about medication errors are reported voluntarily by all classes of care providers (reporting system). The effectiveness of this system depends on the quantity and quality of data reported. Quality in particular refers to data not just about serious errors that cause patient errors but also about "near misses" that fail to reach the patient. The latter kind of data enables organizations, in principle, to proactively address root causes of errors in a blame-free manner.

Data from the reporting system is disseminated to the appropriate people in a timely manner (information-sharing system). The effectiveness of this system depends on the proportion of people involved directly/indirectly with medication delivery who receive the information within a reasonable time from the occurrence of errors. When a greater proportion of people receive the information, awareness about the magnitude of the problem increases and, in turn, encourages further reporting. This also increases the involvement of different classes of providers in analyzing the data.

Information about errors is used by frontline care providers to identify root causes and initiate corrective actions (problem solving system). The effectiveness of this system depends on the ability of care providers to diagnose underlying process issues (in other words go beyond assigning individual blame in identifying causes), design appropriate corrective actions, and implement these actions. When data about errors results in such process improvements, organizational learning is said to occur.

A useful feature of the PRHI experiment is that the participating hospitals implemented a standardized medication 


\section{DATA $\Longrightarrow$ INFORMATION $\longrightarrow$ KNOWLEDGE}



Fig. 1 - Learning chain.

error reporting system called MEDMARX. This database is used by over 775 hospitals and health systems in the U.S. and Canada. Because data is available about national trends in reporting, it is possible to assess how the PRHI hospitals fared relative to other hospital using this database. During the past 3 years PRHI hospitals together reported over 17,000 medication errors, a number significantly higher than baseline reporting levels nationally. Moreover, a greater proportion of errors reported by these hospitals were "near misses". In other words, compared to other hospitals nation-wide that use the MEDMARX system, PRHI hospitals reported a higher quantity as well as quality of errors. But it remains less clear whether this increase in reporting led to increased information-sharing and problem solving and therefore to process improvements that were directly traceable to voluntarily reported errors.

\subsection{Limitations of current research}

Despite a system-wide recognition that change must be affected at all levels of healthcare, research to date on such changes is plagued by two main issues. One, studies addressing the importance of voluntary reporting have lacked requisite empirical support. It is still largely unclear as to how the rates of voluntary errors change over time and what the organizational or structural contributions to these longitudinal effects might be. The present study examines fundamental questions regarding how organizations respond to reports of medication error in hospitals, namely: Does the voluntary nature of reporting encourage error documentation over time? What types of errors are reported?

Two, research has focused mainly on the occurrence (e.g., an error was reported) and has not made an effective distinction between the types of medical errors. Those studies that have attempted such a comparison have done so with largely unstandardized data. Hayard and Hofer [29] underscore the importance of definitional consensus, pointing out that failing to distinguish between error classifications and/or having unstandardized definitions of what a "deadly" error is may lead to inflated estimates of occurrence. This study overcomes the deficits of previous research by utilizing standardized data from multiple healthcare organizations.
Third although the importance of tracking medical errors has been recognized, little research has focused on probing the changes in the patterns of these errors over time. Simulation permits the systematic study of stability and changes over time and can thus provide critically needed evaluations of the courses, causes and consequences of medical errors.

In this study we describe a computer simulation model that can be used to explore organizational changes that are required to improve patient safety based on a medication error reporting system. The model is used to illustrate the fact that patient safety initiatives require more than clinical initiatives. In order to be successful, these initiatives must be designed and implemented through organizational support structures and institutionalized through enhanced education, training, and implementation of information technology that improves work force capabilities [30,31]. For example, Brigham and Women's Hospital was able to reduce the adverse drug event (ACE) rate in the intensive care unit (ICU) by two-thirds by implementing information technology and by having clinical pharmacists participate in patient care with the ICU team [32].

However, information technology implemented to reduce errors may have unintended consequences. One study of the implementation of a computerized physician order entry (CPOE) system in a hospital found that new errors were made in order entry and had to be corrected by pharmacy staff to prevent harm to patients [33]. A second study of a hospital CPOE system found that the system actually facilitated the risk of 22 types of medication errors [34]. Examples of errors include fragmented computer displays of the patient's medications, pharmacy inventory displays that were mistaken as dosage guidelines, and inflexible ordering formats that facilitated double dosing and incompatible orders.

\section{Methods}

\subsection{Data collection}

In order to collect national standardized information on medication errors, the MEDMARX program was created to facilitate 
the collection of information essential to understanding the causes of errors and the development of system-based solutions. The program has been implemented by 775 hospitals and health systems in the U.S. and Canada since 1998. Data from the summary report for 1999-2003 were used to construct the simulation model [22].

In an effort to determine whether hospitals working collectively to report medical errors can improve patient safety, a coalition was formed consisting of 44 hospitals. These hospitals implemented a voluntary retrospective medication error reporting system, the MEDMARX system. Data from these hospitals were used to validate the model.

Table 1 shows the number and types of errors reported for five quarters by the PRHI hospitals. Errors are classified as circumstances that have the potential to cause an error to occur (category A); as errors that do not cause harm to the patient (categories B-D); and as errors that result in harm to or the death of a patient (categories E-I).

\subsection{Simulation model}

A computer simulation model was constructed in order to model medication error reporting systems and organizational changes needed to improve patient safety. STELLA was used to create the model represented in Fig. 2. STELLA is a software package based on the field of systems dynamics. The simulation package permits investigators to apply concepts from feedback control theory to social systems [35].

The model consists of two stages. In stage 1, medication errors are generated based on a normal distribution of med-
Table 1 - Number and type of errors reported over time

by the coalition of hospitals

Type of error

Number (\%)

Category A: circumstances or events that have the capacity to cause error

Category B: an error occurred but the error did not reach the patient

Category C: an error occurred that reached the patient but did not cause patient harm

Category D: an error occurred that reached the patient and required monitoring to confirm that it resulted in no harm to the patient and/or required intervention to preclude harm

Category E: an error occurred that may have contributed to or resulted in temporary harm to the patient and required intervention

Category F: an error occurred that may have contributed to or resulted in temporary harm to the patient and required initial or prolonged hospitalization

Category G: an error occurred that may have contributed to or resulted in permanent patient harm

Category H: an error occurred that required intervention necessary to sustain life.

Category I: an error occurred that may have contributed to or resulted in the patient's death

Total reported medication errors

$1189(5.00)$

$10793(46.00)$

$9622(41.00)$

$1435(6.10)$

$347(1.50)$

$60(0.30)$

$6(<0.001)$

$12(<0.001)$

$2(<0.001)$

$16026(100)$

ication doses and medication errors. Errors are of two types: errors that do not harm patients (categories A-D) and errors that harm the patient (categories E-I). Reporting rates for each type of error are assumed to increase over time according to

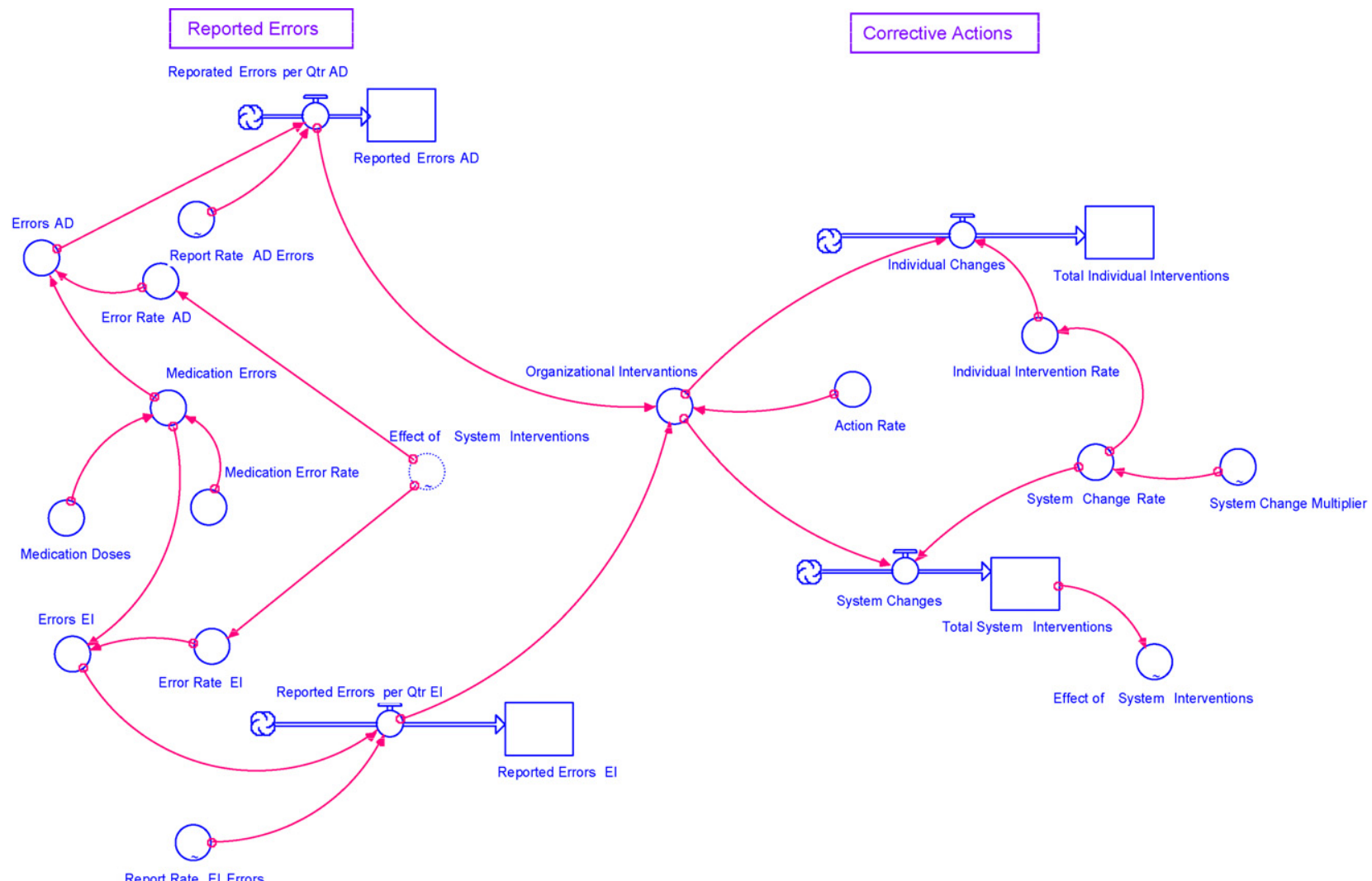

Fig. 2 - Hospital medication error reporting system. 
graphical functions. The rate of increase is based on summary data reported through the MEDMARX system.

The second stage of the model generates actions taken by the health care organization as a result of the reported errors. According to the MEDMARX summary data, health care organizations initiated interventions in response to reported errors only $48 \%$ of the time. This rate was used in the model. The organization can take two types of actions. The first type of action that can be taken by a health care organization in response to reported errors is individual interventions. These interventions may involve informing the staff who made the error, the patient's physician and/or the patient who was affected by the error. Staff education and training also may be provided as a result of an error. These interventions do not change the culture of the organization and may generally have relatively little effect on future errors.

The second type of action involves system changes that alter the organizational structure [36]. These actions include modifying or implementing computer systems such as computer physician order entry (CPOE) or implementing automatic dispensing devices; modifying policies and/or staffing practices; and/or changing formularies. These actions may result in substantial changes in the organizational culture and practices that may lead to greater patient safety. Examples include computerized systems capable of detecting prescription errors and potential and actual ADEs [37-39]. These systems utilize rules to scan medication orders, laboratory results, etc. A list of alerts concerning potential or actual ADEs is automatically generated for care givers. Another example is the assignment of clinical pharmacists to patient care teams [32].

Individual interventions are far more frequent, constituting $96 \%$ of the actions taken in response to reported errors in the MEDMARX system. System interventions that significantly changed the culture of the organization were taken in only $4 \%$ of the cases where an action was taken in response to a reported error. These intervention rates were incorporated into the model.

The model also incorporates feedback. System interventions can result in practice changes that alter the structure and/or culture of the organization. Such changes can result in fewer medical errors. Studies have demonstrated that organizational interventions can significantly reduce serious medication errors. One study found that a CPOE system that was introduced in a tertiary care hospital reduced medication errors that either resulted in or had the potential to result in ADEs by $55 \%$ [40]. A second intervention in which pharmacists participated on physician rounds in an ICU found a decrease of $66 \%$ in preventable ADEs due to prescribing errors [32].

At the same time high rates of ADEs may persist even after the introduction of a computerized medical record. One study found high rates of ADEs related to drug selection, dosage and monitoring in a VA medical center after adoption of computerized systems that had poorly designed data screens, drug dosing details and minimal decision support rules [41]. Other studies by Ash et al. [33] and Koppel et al. [34] have found that $\mathrm{CPOE}$ can facilitate certain types of medication errors. Parameters used in the model are shown in Table 2. These parameters were derived from the literature and from the MEDMARX Fifth Anniversary Data Report [22]. Several potential interventions were simulated. First, the medication error reporting system
Table 2 - Model parameter estimates

\begin{tabular}{|c|c|}
\hline Parameter & Value \\
\hline Medication doses per period & $\begin{array}{l}\text { Normal distribution } M=8000 \text {, } \\
\text { S.D. }=750\end{array}$ \\
\hline Medication error rate & $\begin{array}{l}\text { Normal distribution } M=0.19 \text {, } \\
\text { S.D. }=0.05\end{array}$ \\
\hline Error rate no harm & 0.985 \\
\hline Error rate harm & 0.015 \\
\hline Report rate 1 & Graphical function of time \\
\hline Report rate 2 & Graphical function of time \\
\hline $\begin{array}{l}\text { Rate of overall organizational } \\
\text { interventions }\end{array}$ & 0.48 \\
\hline $\begin{array}{l}\text { Rate of individual interventions } \\
\text { (baseline) }\end{array}$ & 0.96 \\
\hline $\begin{array}{l}\text { Rate of systems interventions } \\
\text { (baseline) }\end{array}$ & 0.04 \\
\hline $\begin{array}{l}\text { Effect of system interventions on } \\
\text { error rates }\end{array}$ & Graphical function of time \\
\hline System change multiplier & Graphical function of time \\
\hline
\end{tabular}

was simulated under baseline conditions. The results were validated against data reported by the 44 hospitals in Pennsylvania. Next, intervention 1 examined the effects of introducing a basic computerized physician order entry system with minimal decision support for the medication prescribing process. Intervention 2 was performed assuming the implementation of a CPOE system with decision support. Intervention 3 assumed that a clinical pharmacist participated on physician rounds and reviewed all medication orders written. Intervention 4, assumed an organizational commitment to undertake root-cause analyses and system changes to prevent future errors from occurring.

\section{Results}

The model was used to simulate medication error reporting in a typical hospital over 12 quarters. The model predicts the number of medication errors reported by type and organizational actions taken as a result of reported errors. Fig. 3 shows the results over the 12 quarters. As can be seen from the graphs, the number of errors reported increased over time. This suggests that, as a hospital gains experience with the error reporting system, health care providers report a greater proportion of errors that occur.

The model predicts that over 12 quarters a total of 1632 errors will be reported. Table 3 shows the predicted actions taken by the hospital in response to reported errors. A total of 1632 errors were reported over the 12 quarters simulated by the model. The baseline model predicts that only $2 \%$ of the reported errors led to system changes to prevent future errors from reoccurring.

In order to validate the model, the model predictions for the first five quarters were compared to actual data from the Pittsburgh regional coalition of 44 hospitals (see Table 4). Predicted values are quite close to the actual number of reported errors.

Studies have indicated that system changes that involve the implementation of information technology such as computerized physician order entry (CPOE) or staff changes such 


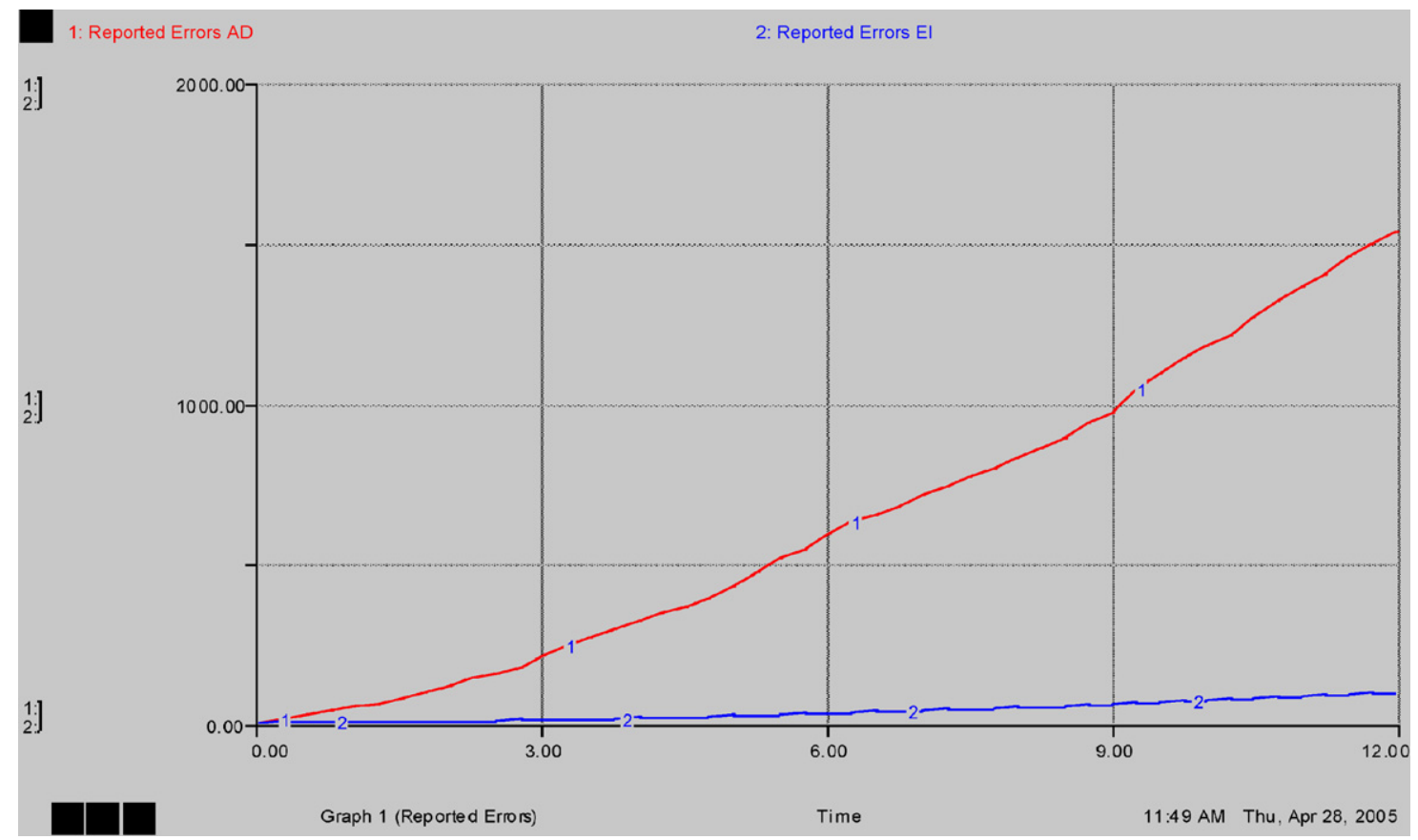

Fig. 3 - Reported medication errors over 12 quarters.

as assigning clinical pharmacists to participate on physician hospital rounds and review medication errors can reduce the incidence of significant errors that could potentially harm patients. Four interventions were simulated and compared to

\section{Table 3 - Organizational actions in response to reported} errors

\begin{tabular}{lcc}
\hline Quarter & Individual changes & System changes \\
\hline 1 & 23 & 1 \\
2 & 31 & 1 \\
3 & 45 & 2 \\
4 & 54 & 2 \\
5 & 51 & 3 \\
6 & 81 & 3 \\
7 & 60 & 2 \\
8 & 57 & 3 \\
9 & 67 & 3 \\
10 & 106 & 4 \\
11 & 90 & 3 \\
12 & 87 & 4 \\
Total & 752 & 31 \\
\hline
\end{tabular}

Table 4 - Comparison of predicted and actual reported medication errors

\begin{tabular}{lcc} 
Quarter & $\begin{array}{c}\text { Predicted reported } \\
\text { errors }\end{array}$ & $\begin{array}{c}\text { Actual reported } \\
\text { errors }\end{array}$ \\
\hline 1 & 49 & 51 \\
2 & 69 & 62 \\
3 & 96 & 108 \\
4 & 118 & 117 \\
5 & 111 & 107 \\
Total & 443 & 445 \\
\hline
\end{tabular}

the baseline simulation. The first organizational intervention that was simulated assumed that a CPOE system with minimal decision support capabilities was implemented in the hospital. The second intervention simulated the effect on serious medication errors of implementing a CPOE system with decision support for drug selection, dosing and monitoring. A third simulation examined the results of pharmacists' participation on physician rounds. The fourth simulation represented a situation where pharmacists participated on rounds as well as an organizational commitment to identifying the causes of errors and making system changes to improve patient safety. For each of these simulations the graphical systems effect parameter was modified to reflect published studies that report the effects of the intervention over time on medication errors. For the fourth simulation a multiplier was introduced to reflect organizational learning and a shift over time to root cause analysis of errors and system changes to prevent them from reoccurring. Results are shown in Table 5.

The first intervention simulated the implementation of a CPOE system with minimal decision support. The intervention failed to reduce serious medication errors. In fact, during five quarters medication errors slightly increased over the baseline.

The second intervention, introduction of a CPOE with decision support, resulted in a $21 \%$ reduction on average of serious medication errors from 130 to 103 per quarter. The third intervention involving the inclusion of a clinical pharmacist on physician rounds had a larger effect on medication errors. The model predicted a reduction of $27 \%$ in errors that could have resulted in ADEs. Over the 12 quarters a total of 453 medication errors could have been prevented by this intervention.

The final intervention that involves organizational learning based on the error reporting system and a commitment to system changes to prevent future errors had the largest effect. 
Table 5 - Estimated number of serious medication errors that could have resulted in adverse drug effects by quarter

\begin{tabular}{|c|c|c|c|c|c|}
\hline Quarter & Baseline & Intervention 1 & Intervention 2 & Intervention 3 & Intervention 4 \\
\hline 1 & 162 & 161 & 130 & 126 & 154 \\
\hline 2 & 118 & 117 & 104 & 131 & 146 \\
\hline 3 & 145 & 124 & 118 & 91 & 109 \\
\hline 4 & 120 & 141 & 129 & 88 & 64 \\
\hline 5 & 135 & 150 & 114 & 84 & 74 \\
\hline 6 & 121 & 118 & 109 & 96 & 58 \\
\hline 7 & 124 & 133 & 91 & 79 & 63 \\
\hline 8 & 116 & 100 & 110 & 77 & 42 \\
\hline 9 & 134 & 141 & 94 & 88 & 40 \\
\hline 10 & 134 & 112 & 85 & 73 & 29 \\
\hline 11 & 122 & 122 & 80 & 67 & 34 \\
\hline 12 & 127 & 145 & 71 & 104 & 39 \\
\hline Total & 1557 & 1566 & 1235 & 1104 & 852 \\
\hline Average & 130 & 131 & 103 & 92 & 71 \\
\hline
\end{tabular}

By the end of the 12 quarters the rate of serious medication errors was reduced by almost $70 \%$ compared to the baseline. Over the total period of time serious errors were reduced by $45 \%$.

\section{Discussion}

This study describes a computer simulation model that has been developed to explore the effects of organizational changes in response to a medication error reporting system. The model is based on the premise that successful patient safety initiatives require more than reporting of errors. In order to substantially reduce medication errors that have the potential to harm patients, health care institutions need to routinely share the information on errors with patients and providers, identify root causes of the errors and initiate problem solving and system changes to prevent similar errors from occurring in the future.

The model predicted that the number of medication errors reported by hospital staff would increase over time. This finding was corroborated by data from the Pittsburgh regional coalition of hospitals. However, the baseline model constructed from summary data reported to the MEDMARX system also indicated that organizational actions needed to reduce the risk of future errors occurred less than $48 \%$ of the time and found that $96 \%$ of the actions taken in response to reported errors involved individual staff.

Several potential organizational system interventions were simulated involving the introduction of a CPOE system and pharmacist participation on physician rounds. The model predicted that the implementation of a basic CPOE system would have little effect on the rate of serious medication errors over 12 quarters. The second and third interventions simulated CPOE with decision support and pharmacist participation, both resulted in a significant reduction of medication errors that could harm patients. A fourth simulation assumed that organizational learning occurred in the hospital as errors were reported. Also, it assumed that a commitment was made to increase system changes to prevent the same types of errors from reoccurring in the future. The model predicted that in this case the intervention would reduce the medication error rate by almost $70 \%$ over time and prevent 705 serious errors that could have harmed patients from occurring.

The study has several limitations. Error rates and reporting rates were based on published studies. These rates vary considerably from hospital to hospital and over time as an analysis of reporting rates for the 44 hospitals in Pennsylvania indicated. Also, the same analysis indicated that error reporting rates increased over time. We tried to take this into account by representing the medication error rate as a normal distribution and error reporting rates in the model as a function of time.

The CPOE interventions that were simulated also were based on parameter estimates from published studies. However, CPOE systems vary considerably in the way they are implemented. They may function largely as stand alone systems or interface with other systems (e.g., laboratory, pharmacy, patient care). They may incorporate decision support for prescribing, dosing, etc. As a result the effect of these interventions will vary greatly from hospital to hospital depending upon the specific features of the CPOE system.

\section{Conclusions}

Individual mistakes alone are not the cause of medication errors. Errors occur within an organizational context characterized by a weak safety culture, inadequate operational practices and protocols, poor training and communication $[25,42]$. While error reporting systems and information technology can partially correct some of these problems, they may not completely solve them. The results of this study indicate that a significant number of medication errors continued to occur even after a medication error reporting system was implemented in the hospital. Furthermore, simulation indicated that adding information technology and changing rounds to include a clinical pharmacist only reduced errors by $20-27 \%$. A significant reduction in medication errors only occurred when the simulated hospital put into place a strategy of root cause analysis when an error was reported and made system changes to prevent this type of error from reoccurring in the future. In this case the model predicted a reduction of almost $70 \%$ in the error rate over time. 
In conclusion organizational actions taken as a result of errors indicate how aggressive the institution is in responding to reported errors. An effective patient safety program facilitates reporting of errors and learning from these errors. Organizational actions that only affect individual staff are likely to have little effect in reducing future errors. Organizational or system changes could result in sustained changes in the organizational culture and practices if implemented properly. These changes could lead to a significant improvement in patient safety.

The results of this study suggest that there is a mismatch between patient safety goals and hospital actions to reduce the risk of future medication errors. Hospitals increasingly seem to recognize the need to implement voluntary error reporting systems in order to gather information needed to reduce errors. At the same time, they fail to initiate organizational changes that are needed to improve patient safety. Typically hospital efforts are narrowly focused on communicating information about errors to health care providers. More than the implementation of voluntary reporting systems is required. Significant reductions in errors will also require that organizational changes, consistent with the improvement of patient safety, be carefully institutionalized and integrated into long-term plans.

\section{Acknowledgments}

This research project was supported in part by a grant from the Regenstrief Institute for Health Care Engineering. This project was also supported by the Agency for Healthcare Research and Quality (U18 HS1 1926-02). The Pittsburgh Regional Healthcare Initiative receives additional support from the Centers for Disease Control and Prevention, the Centers for Medicare and Medicaid Services, the Robert Wood Johnson Foundation, the Jewish Healthcare Foundation, and other local foundations, health plans, and employers.

\section{REFERENCES}

[1] K.T. Kohn, J.M. Corrigan, M.S. Donaldson (Eds.), To Err is Human: Building a Safer Health System., Institute of Medicine, National Academy Press, Washington, DC, 2001.

[2] D.P. Phillips, C.C. Bredder, Morbidity and mortality from medical errors: an increasingly serious public health problem, Ann. Rev. Pub. Health 23 (2002) 135-150.

[3] J. Lazarou, B.H. Pomeranz, P.N. Corey, Incidence of adverse drug reactions in hospitalized patients: a meta-analysis of prospective studies, J. Am. Med. Assoc. 279 (1998) 1200-1205.

[4] K.N. Barker, E.A. Flynn, G.A. Pepper, D.W. Bates, R.L. Mikeal, Medication errors observed in 36 health care facilities, Arch. Intern. Med. 162 (2002) 1897-1903.

[5] Institute of Medicine, Crossing the Quality Chasm: A New Health System for the 21st Century. National Academy Press, Washington, DC, 2001.

[6] L.L. Leape, Reporting of adverse events, New England J. Med. 347 (20) (2002) 1633-1638.

[7] D.J. Cullen, D.W. Bates, S.D. Small, et al., The incident reporting system does not detect adverse drug events: a problem for quality improvement, J. Qual. Im. 21 (1995) 541-548.
[8] L.L. Leape, D.M. Berwick, Five years after to err is human: what have we learned, J. Am. Med. Assoc. 293 (2005) 2384-2390.

[9] C.E. Billings, Some hopes and concerns regarding medical event-reporting systems: lessons from the NASA aviation safety reporting system, Arch. Pathol. Lab. Med. 122 (3) (1998) 214-215.

[10] Veterans Administration Patient Safety reporting System (PSFS), http://www.psrs.arc.nasa.gov/.

[11] D. Mears, S.V. White, P. James, Bagian on patient safety initiative, J. Health Care Qual. 24 (15-16) (2002) 24.

[12] Institute for Safe Medication Practices Medication Error Program, http://www.ismp.org/pasgesd/communication.asp.

[13] P.J. Pronovost, A.W. Wu, T. Dorman, L. Morlock, Building safety into ICU care, J. Crit. Care 17 (2002) 78-85.

[14] H.S. Mekhjian, T.D. Bentley, A. Ahmad, G. Harsh, Development of a web-based event reporting system in an academic environment, J. Am. Med. Inform. Assoc. 11 (1) (2004) 11-18.

[15] D.H. Fernald, W.D. Pace, et al., Event reporting to a primary care safety reporting system: A report from the ASIPS collaborative, Ann. Family Med. 2 (2004) 327332.

[16] A. Wu, P. Pronovost, L. Morlock, ICU incident reporting systems, J. Crit. Care 17 (2002) 86-94.

[17] J. Kim, D.W. Bates, Results of a survey on medical error reporting systems in Korean hospitals, Int. J. Med. Inform. 75 (2) (2006) 148-155.

[18] H. Furukawa, H. Bunko, F. Tsuchiya, K. Miyamoto, Voluntary medication error reporting program in a Japanese national university hospital, Ann. Pharmacother. 37 (11) (2003) 1716-1722.

[19] F. Le Duff, S. Daniel, B. Kamendje, P. Le Beux, R. Duvauferrier, Monitoring incident report in the healthcare process to improve quality in hospitals, Int. J. Med. Inform. $74(2-4)$ (2005) 111-117.

[20] C.G. Holzmueller, P.J. Pronovost, F. Dickman, D.A. Thompson, A.W. Wu, L.H. Lubomski, M. Fahey, D.M. Steinwachs, L. Engineer, A. Jaffrey, Creating the web-based intensive care unit safety reporting system, J. Am. Med. Inform. Assoc. 12 (2) (2005) 130-139.

[21] C.A. Sirio, K.T. Segel, D.J. Keyser, E.I. Harrison, J.C. Lloyd, R.J. Weber, C.A. Muto, D.G. Webster, V. Pisowicz, K.W. Feinstein, Pittsburgh regional healthcare initiative: a systems approach for achieving perfect patient care, Health Affairs 22 (5) (2003) 157-165.

[22] R.W. Hicks, J.P. Santell, D.D. Cousins, R.L. Williams, MEDMARX Fifth Anniversary Data Report: A Chartbook of 2003 Findings and Trends 1999-2003, 2004.

[23] National Coordinating Council for Medication Error Reporting and Prevention, What is a Medication Error?, 1998.

[24] V.L. Patel, L.M. Currie, Clinical cognition and biomedical informatics: Issues of patient safety, Int. J. Med. Inform. 74 (11-12) (2005) 559-561.

[25] R.L. Simpson, Managing the three 'P's to improve patient safety: Nursing administration's role in managing information technology, Int. J. Med. Inform. 73 (7-8) (2004) 111-117.

[26] R. Ramanujam, D.J. Keyser, C.A. Sirio, Missing: The logic of organizational change in patient safety initiatives, Unpublished manuscript, 2004.

[27] R. Cook, M. Render, D. Woods, Gaps in the continuity of care and progress on patient safety, Br. Med. J. 320 (2000) 791-794.

[28] J.G. Anderson, A system's approach to preventing adverse drug events, in: S. Krishna, E.A. Balas, S.A. Boren (Eds.), Information Technology Business Models for Quality Health Care: An EU/US Dialogue, IOS Press, The Netherlands, 2003, pp. 95-102. 
[29] R.A. Hayard, T.P. Hofer, Estimating hospital deaths due to medical errors: preventability is in the eye of the reviewer, J. Am. Med. Assoc. 286 (4) (2001) 415-420.

[30] J.G. Anderson, S.J. Jay, M. Anderson, T.J. Hunt, Evaluating the capability of information technology to prevent adverse drug events: A computer simulation approach, J. Am. Med. Inform. Assoc. 9 (2002) 479-490.

[31] J.G. Anderson, Information technology for detecting medication errors and adverse drug events, Expert Opin. Drug Saf. 3 (5) (2004) 449-455.

[32] L.L. Leape, D.J. Cullen, M.D. Clapp, E. Burdick, H.J. Demonaco, J.I. Erickson, D.W. Bates, Pharmacist participation on physician rounds and adverse drug events in the intensive care unit, J. Am. Med. Assoc. 272 (1999) 267-270.

[33] J.S. Ash, M. Berg, E. Coiera, Some unintended consequences of information technology in health care: the nature of patient care information system-related errors, J. Am. Med. Inform. Assoc. 11 (2) (2004) 104-112.

[34] R. Koppel, J.P. Metlay, A. Cohen, B. Abaluck, A.R. Localio, S.E. Kimmel, B.L. Strom, Role of computerized physician order entry systems in facilitating medication errors, J. Am. Med. Assoc. 293 (2005) 1197-1203.

[35] B. Hannon, M. Ruth, Dynamic Modeling, Springer-Verlag, New York, 1994.

[36] R. Behal, Organizational development framework for transformational change in patient safety: a guide for hospital senior leaders, in: B.J. Youngberg, M.J. Hatlie (Eds.),
The Patient Safety Handbook, Jones and Bartlett Publishers, Boston, 2004.

[37] D.C. Classen, S.L. Pestotnik, R.S. Evans, J.P. Burke, Computerized surveillance of adverse drug events in hospital patients, J. Am. Med. Assoc. 266 (1991) 28472851.

[38] A.K. Jha, G.J. Kuperman, J.M. Teich, L. Leape, B. Shea, E. Rittenberg, E. Burdick, D.L. Seger, M.V. Vliet, D.W. Bates, Identifying adverse drug events: development of a computer-based monitor and comparison with chart review and stimulated voluntary report, J. Am. Med. Inform. Assoc. 5 (1998) 305-314.

[39] A. Oliven, I. Michalake, D. Zalman, E. Dorman, D. Yeshurun, M. Odeh, Prevention of prescription errors by computerized, on-line surveillance of drug order entry, Int. J. Med. Inform. 74 (5) (2005) 377-386.

[40] D.W. Bates, L.L. Leape, D.J. Cullen, N. Laird, L.A. Petersen, J.M. Teich, E. Burdick, M. Hickey, S. Kleefield, B. Shea, M.V. Vliet, D.L. Seger, Effect of computerized physician order entry and a team intervention on prevention of serious medication errors, J. Am. Med. Assoc. 280 (1998) 13111316.

[41] J.R. Nebeker, J.M. Hoffman, C.R. Weir, C.L. Bennett, J.F. Hurdle, High rates of adverse drug events in a highly computerized hospital, Arch. Intern. Med. 165 (2005) 1111-1116.

[42] L. Donaldson, Keep the patients safe, Qual. World 29 (2) (2003) 10-12. 